University of Nebraska - Lincoln

DigitalCommons@University of Nebraska - Lincoln

August 1999

\title{
Two-dimensional nuclear magnetic resonance correlation spectroscopy at zero field
}

\author{
Ming-Yuan Liao \\ University of Nebraska - Lincoln \\ Gerard S. Harbison \\ University of Nebraska - Lincoln, gharbison1@unl.edu
}

Follow this and additional works at: https://digitalcommons.unl.edu/chemistryharbison

Part of the Chemistry Commons

Liao, Ming-Yuan and Harbison, Gerard S., "Two-dimensional nuclear magnetic resonance correlation spectroscopy at zero field" (1999). Gerard Harbison Publications. 1.

https://digitalcommons.unl.edu/chemistryharbison/1

This Article is brought to you for free and open access by the Published Research - Department of Chemistry at DigitalCommons@University of Nebraska - Lincoln. It has been accepted for inclusion in Gerard Harbison Publications by an authorized administrator of DigitalCommons@University of Nebraska - Lincoln. 


\title{
Two-dimensional nuclear magnetic resonance correlation spectroscopy at zero field
}

\author{
Ming-Yuan Liao ${ }^{\text {a) }}$ and Gerard S. Harbison \\ Department of Chemistry, 508 Hamilton Hall, University of Nebraska at Lincoln, Lincoln, \\ Nebraska 68588-0304
}

(Received 20 April 1999; accepted 21 May 1999)

\begin{abstract}
Three methods for two-dimensional correlation nuclear magnetic resonance spectroscopy at zero field are discussed. All three involve coherence transfer via longitudinal polarization, double quantum coherence, or both in parallel. The double quantum pulse sequences exploit the spinor property of spin states. These sequences have been applied to connected $\Delta m=1$ transitions, as well as for the indirect detection of forbidden or nearly forbidden $\Delta m>1$ transitions. (c) 1999 American Institute of Physics. [S0021-9606(99)00331-1]
\end{abstract}

\section{INTRODUCTION}

Despite their success in high-field nuclear magnetic resonance (NMR), extension of multidimensional Fourier transform methods to zero-field NMR has been limited. There are several legitimate reasons for this; principally, high-field spectra are often complex, with literally thousands of resonance signals; in such systems, two-dimensional methods are necessary both to increase the resolution and provide spectral assignments. In contrast, zero-field spectra are often very simple, both because of the paucity of chemically distinct species in the material, and because there is usually only a single significant term in the spin Hamiltonian, resulting in a lack of spectral multiplicity.

Nonetheless, there are occasions when multidimensional techniques can be usefully applied at zero field. The first such applications ${ }^{1-3}$ were directed at extracting the asymmetry parameter, ordinarily unavailable for spin $3 / 2$ nuclei at zero field. For spins $5 / 2$ and higher, $\eta$ can be obtained from the ratios of two or more spectral frequencies observable for each species. However, in samples with several distinct quadrupolar species, such as partially deuterated amine and amino acid hydrohalides, ${ }^{4}$ connected transitions must be correctly assigned to extract this information. This was the purpose of our original nuclear quadrupolar correlation experiment. ${ }^{5}$ Since then, we have also applied the experiment to inhomogeneously broadened systems, since in such systems the quadrupolar frequencies are strongly correlated. The resulting removal of this broadening has allowed us to estimate the hexadecapolar interaction for ${ }^{127} I$ In cadmium iodide with high accuracy. ${ }^{6}$

Figure 1 correlates the energy levels and transitions for a spin $5 / 2$ nucleus at zero field with the high-field energy levels. At zero field, the high-field eigenstates, labeled by the quantum number $m$ associated with the $z$ component of the angular momentum, become twofold degenerate, with the $\pm m$ states having the same energy. There are two connected single-quantum transitions at frequencies $\nu_{1}$ and $\nu_{2}$ and one double-quantum transition at $\nu_{d q}=\nu_{2}+\nu_{1}$. For an axially

\footnotetext{
${ }^{a}$ Current Address: Department of Applied Chemistry, National Chi Nan University, Puli, Taiwan, Republic of China.
}

symmetric electric field gradient $\nu_{2}=2 \nu_{1}$, and the eigenstates are identical to the high-field eigenstates. If the electric field gradient is not axially symmetric, the ratio between the two single-quantum frequencies decreases until it reaches a value of 1 for an asymmetry parameter $\eta=\left(\nabla E_{y y}\right.$ $\left.-\nabla E_{x x}\right) / \nabla E_{z z}=1$, and concomitantly there is some mixing of the high-field eigenstates, the $m= \pm 5 / 2$ states being partially mixed with $m= \pm 1 / 2$ and the $m= \pm 3 / 2$ state with $m$ $=\mp 1 / 2$. For simplicity's sake, we shall henceforth refer to the degenerate states corresponding to quantum numbers $\pm m$ simply as $m$.

The original NQ-COSY experiment used a strategy common to many high-field NMR experiments: the first transition was excited, allowed to evolve, one component of the transverse magnetization returned to the $z$ direction, and then the second (connected) transition excited and observed. This strategy has the virtue of simplicity, and in many systems at high field it is the only alternative. It has however, one major drawback: it wastes half of the initial nuclear polarization.

There exists a second method of transferring coherence between connected transitions: via the double-quantum coherence. In this paper, we explore alternative strategies for correlation spectroscopy employing this route of polarization transfer, weigh the advantages of the two transfer pathways, and consider hybrid methods employing both.

\section{MATERIALS AND METHODS}

Antimony(III) chloride and bismuth(III) chloride are obtained from Johnson Matthey Electronics and Fluka. The quadrupole resonance frequencies of ${ }^{121} \mathrm{Sb}(I=5 / 2),{ }^{123} \mathrm{Sb}$ $(I=7 / 2)$, and ${ }^{209} \mathrm{Bi}(I=9 / 2)$ in antimony(III) chloride and bismuth(III) chloride are obtained from the literature. ${ }^{7,8}$

All experiments were carried out using a home-built pulsed double-resonance solid-state NMR spectrometer at zero field. Since it was not necessary in any of these experiments simultaneously to irradiate one transition while observing a second, no attempt was made to frequency isolate the two probe resonances; rather, the radio-frequency output from the two spectrometer channels was combined before final amplification, using a single linear high-power amplifier, and the output sent to the probe circuit shown in Fig. 2. This circuit provides two probe resonances which can be 
(a)

(b)

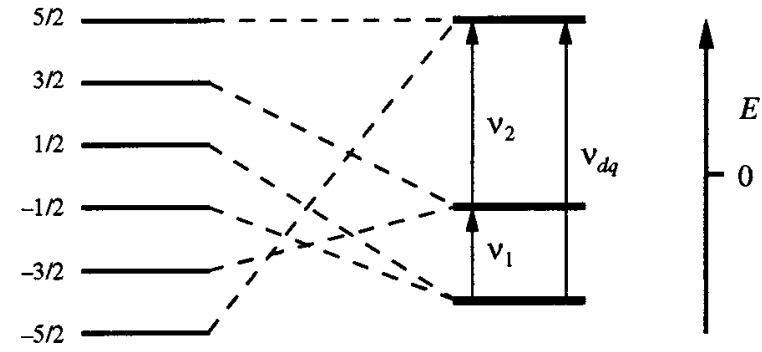

FIG. 1. Energy levels and coherences of a spin-5/2 nucleus (a) at high field, with no quadrupole coupling present (b) at zero field.

tuned largely independently of each other, and which can be matched individually to $50 \Omega$ using the matching inductor $L_{3}$ and by adjusting the inductive coupling between $L_{1}, L_{2}$, and $L_{3}$. The sample coil in this probe configuration is usually $L_{1}$

The samples were packed into a glass tube of whose dimensions are $10 \times 40 \mathrm{~mm}$, and the probe tuned to the resonance frequencies of two connected spin transitions of the sample. The temperature was maintained at $24.5 \pm 0.5^{\circ} \mathrm{C}$ by air cooling.

\section{RESULTS AND DISCUSSION}

\section{Longitudinal coherence transfer}

Coherence transfer via longitudinal magnetization can best be approached conceptually from the simpler nutation experiment diagrammed in Fig. 3(a). In this experiment, $\nu_{1}$ and $\nu_{2}$ correspond to the frequencies of two connected NMR transitions. Pulses of variable length $t_{1}$ is applied to the transition at $\nu_{1}$, causing the polarization, initially aligned along the $z$ direction, to process into the transverse direction, which we can call $x$. As a result, the residual polarization of a single crystallite is proportional to $I_{0} \cos \omega t_{1}$, with $\omega$, the nutation frequency, being a function of the nuclear gyromagnetic ratio, the radio-frequency field strength, the quadrupolar asymmetry parameter, and the crystallite orientation. If the two transitions corresponding to $\nu_{1}$ and $\nu_{2}$ are connected, then a pulse on the connected transition at $\nu_{2}$ will give an observable signal dependent on the residual $z$ polarization of the first transition. The first pulse on the (5/2-3/2) transition of the ${ }^{123} \mathrm{Sb}$ nucleus changes the population difference between these two states. This increases the population difference between the $3 / 2$ and the $1 / 2$ state above that of thermal equi-

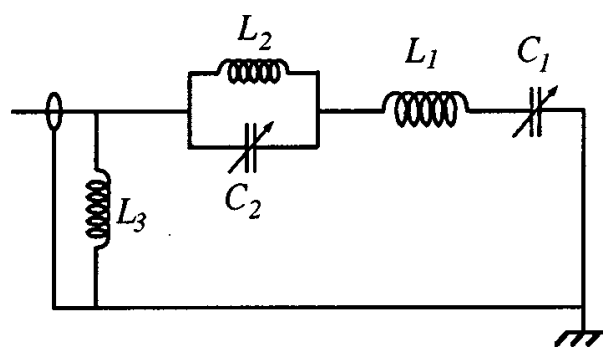

FIG. 2. Double resonance probe circuit used in this work. $C_{1}$ and $C_{2}$ are vacuum variable capacitors (Jennings, $15 \mathrm{kV}$ breakdown voltage, 1.5-30 $\mathrm{pF})$, while $L_{1}-L_{3}$ are inductors. (a)

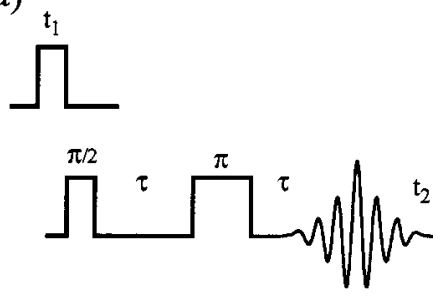

(b)

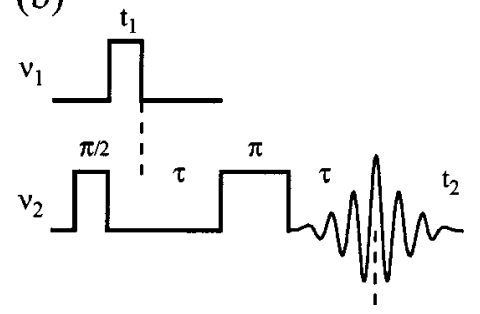

FIG. 3. Pulse sequences for nutation spectroscopy at zero field. (a) Longitudinal nutation experiment. (b) Spinor nutation experiment.

librium. If, subsequently, a spin echo pulse sequence is applied to observe the $(3 / 2-1 / 2)$ transition, the effect of the first pulse length on the intensity of the second transition can be observed.

This is demonstrated in Fig. 4(a), in which we show the intensity of the $(3 / 2-1 / 2)$ transition of the ${ }^{123} \mathrm{Sb}$ nucleus in antimony(III) chloride at an observation frequency of $\nu_{2}$ $=37.400 \mathrm{MHz}$, as a function of the length of a pulse on the
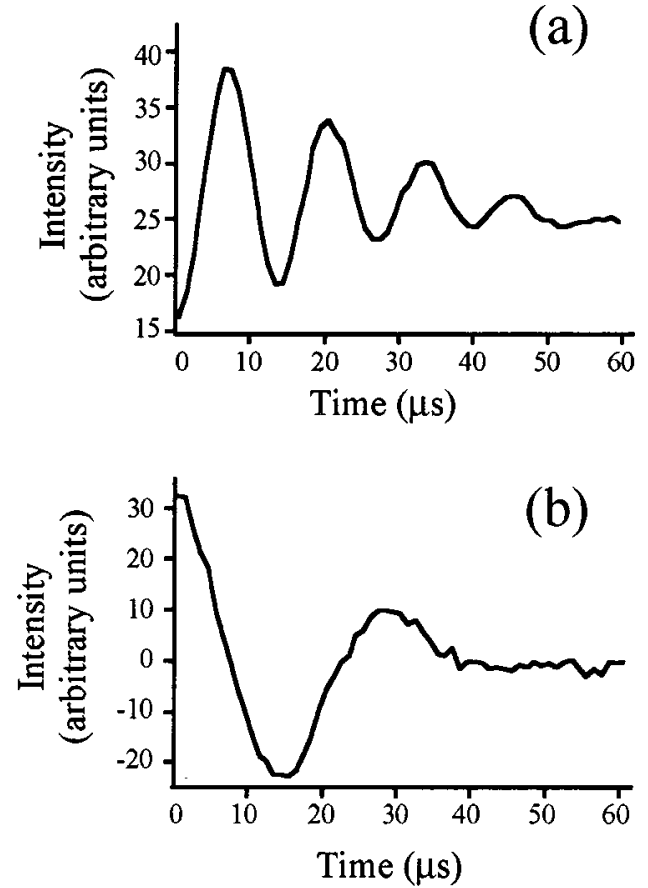

FIG. 4. (a) Intensity of the $3 / 2-1 / 2$ transition of the ${ }^{123} \mathrm{Sb}$ signal in antimony(III) chloride, as a function of the time of nutation of the 5/2-3/2 transition, obtained using the pulse sequence in Fig. 2(a). (b) Intensity of the $3 / 2-1 / 2$ transition of the ${ }^{123} \mathrm{Sb}$ signal in antimony(III) chloride, as a function of the time of nutation of the 5/2-3/2 transition, obtained using the pulse sequence in Fig. 2(b). Both spectra are obtained with transmitter frequencies $\nu_{1}=67.8 \mathrm{MHz}$ and $\nu_{2}=37.4 \mathrm{MHz}$, close to the respective resonance frequencies of the (5/2-3/2) and (3/2-1/2) transitions. 


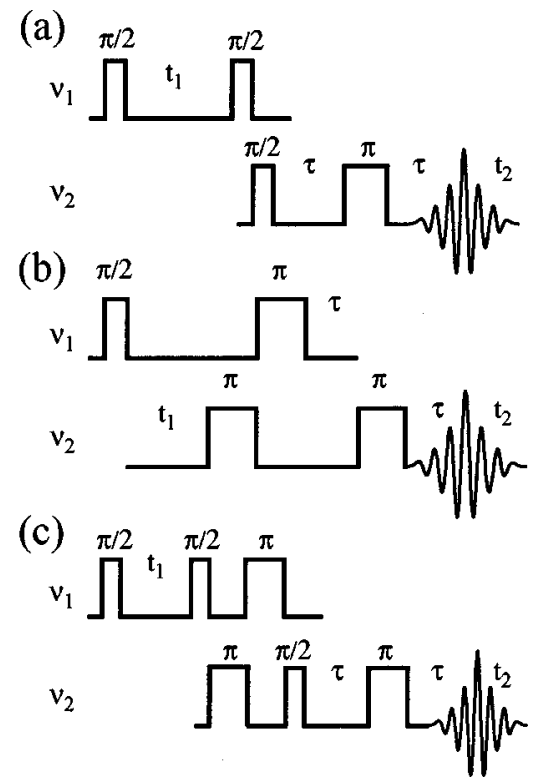

FIG. 5. Pulse sequences for two-dimensional NMR correlation spectroscopy at zero field. (a) NQ-COSY. (b) NQ-SCOSY. (c) NQ-MSCOSY.

(5/2-3/2) transition. The nominal nutation frequency is 65 $\mathrm{kHz}$, however, because the precession frequency of individual crystallites in a powder NQR sample depends on the orientation of the electric field gradient tensor relative to the coil axis, ${ }^{2,9}$ this is merely the predominant value in the powder. The maximum enhancement measured for the $(3 / 2-1 / 2)$ transition is 2.64 , corresponding to a nominal $\pi$ pulse on the $5 / 2-3 / 2$ transition, which is close to the maximum possible value for a single crystal of $\left(\nu_{1}+\nu_{2}\right) / \nu_{2}=2.81$.

The nutation experiment, which merely demonstrates polarization transfer, can be turned into a coherence transfer experiment by replacing the variable pulse $t_{1}$ by two nominal $\pi / 2$ pulses separated by a variable delay $t_{1}$, as shown in Fig. 5(a). Since this pulse sequence has already been published, ${ }^{5}$ it will be discussed only in the most cursory detail. The first $\pi / 2$ pulse creates single quantum coherence corresponding to the (5/2-3/2) transition for the spin system. The singlequantum coherence evolves during the $t_{1}$ period and acquires a phase $\phi=\omega_{1} t_{1}$. Applying a second $\pi / 2$ pulse on the same $(5 / 2-3 / 2)$ transition rotates the component out-of-phase with the second pulse back along the $z$ or $-z$ direction, leaving the in-phase component unaffected. The $z$ magnetization of the $(3 / 2-1 / 2)$ transition is therefore now modulated by $\cos \phi$ or $\sin \phi$. The perturbed $z$ polarization can then be sampled using a $\pi / 2-\tau-\pi$ spin echo pulse sequence on the connected transition. Both real and imaginary parts of the transverse magnetization are collected in two separate experiments to generate quadrature information in the first dimension. Other artifacts, such as those arising from the production of double-quantum coherence from the singlequantum magnetization remaining after the second $\pi / 2$ pulse, can also be removed be phase cycling. The spin echo on the $(3 / 2-1 / 2)$ transition is used to avoid dead-time problems and does not complicate the spin dynamics. After processing by the method of States et al., ${ }^{10}$ a two-dimensional spectrum correlating the $(5 / 2-3 / 2)$ and $(3 / 2-1 / 2)$ transitions of
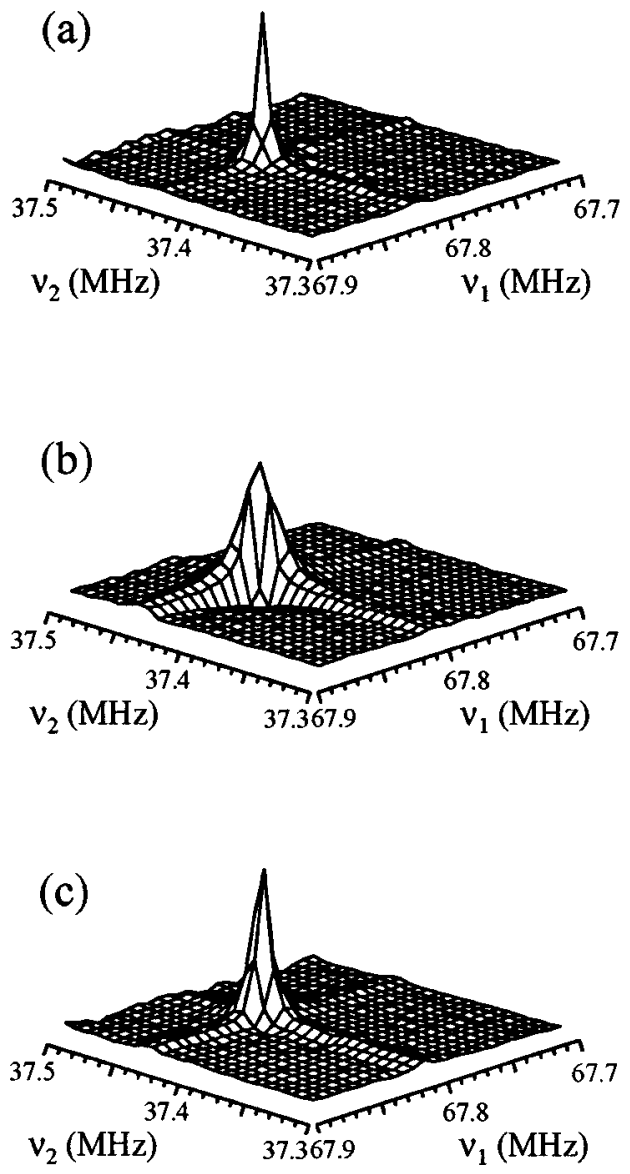

FIG. 6. Two-dimensional zero-field NMR correlation spectrum of the ${ }^{123} \mathrm{Sb}$ nucleus in antimony(III) chloride recorded by (a) the NQ-COSY pulse sequence, (b) the NQ-SCOSY pulse sequence, (c) the NQ-MSCOSY pulse sequence.

${ }^{123} \mathrm{Sb}$ in antimony trichloride was obtained, and is shown in Fig. 6(a). As can be seen, the spectrum obtained has pure phase in both dimensions.

While this sequence has the virtue of simplicity, the Hermiticity of the density matrix means that purely real longitudinal elements, through which coherence is transferred, can only contain half of the (complex) coherence information, and half of the initial magnetization is lost, an inevitability in any sequence which relies on coherence transfer via longitudinal polarization.

\section{Coherence transfer via the double-quantum coherence}

The pulse sequence shown in Fig. 3(b) seems at first glance to be a trivial variation on that in 3(a); the order of the first two pulses is reversed. The rearrangement, however, has profound consequences. Following creation of singlequantum coherence of the $\nu_{2}$ transition by the nominal $\pi / 2$ pulse on that transition, the pulse of variable length $t_{1}$ now converts that coherence into double-quantum coherence between the top and bottom states of the system. Moreover, because the pulse affects only one of the two states involved in the initial coherence, the evolution of that state follows the behavior of a spinor. ${ }^{11}$ The observed signal in the experiment, shown in Fig. 4(b), obtained under identical conditions 
to those used in Fig. 4(a) (except for the rearrangement of the pulse sequence), has a measured nutation frequency of 31.7 $\mathrm{kHz}$, which is approximately half of the nutation frequency measured via the experiment in Fig. 3(a). Complete conversion of the single-quantum signal into the (nearly invisible) double-quantum coherence is achieved with a nominal $\pi$ pulse, inversion of the transition with a $2 \pi$ pulse, and (approximate) regeneration of the original single-quantum coherence only after a $4 \pi$ pulse. Such effects have been observed previously in various forms of magnetic resonance. $^{12,13}$

The double-quantum coherence produced by the $\pi$ pulse, unlike longitudinal magnetization, has a phase associated with it, which can in fact be written as the simple sum of the phase of the single-quantum coherence and that of the $\pi$ pulse. Therefore, in a two-dimensional NMR experiment, it can retain all of the phase information present in the evolved single-quantum coherence at the end of the $t_{1}$ period.

A two-dimensional coherence transfer experiment based on such double-quantum coherence transfer is shown in Fig. 5(b), and we have dubbed this experiment SCOSY (for spinor COSY). A $\pi / 2\left(\nu_{1}\right)-t_{1}-\pi\left(\nu_{2}\right)$ pulse sequence creates single-quantum coherence for the (5/2-3/2) transition, allows it to evolve, and then transfers it to double-quantum (5/2-1/2) coherence, as described above. A second $\pi$ pulse, this time on the (5/2-3/2) transition, converts the doublequantum coherence to single-quantum coherence of the (3/2$1 / 2$ ) transition. This pair of $\pi$ "spinor pulses" therefore has the net effect of transferring the coherence between two connected single-quantum transitions. After the refocusing $\pi$ pulse on the (3/2-1/2) transition, the transferred coherence, which contains phase information acquired during the evolution $t_{1}$ period, is observed during the detection $t_{2}$ period.

Unlike the simple NQ-COSY experiment, SCOSY retains all the initial polarization of the (5/2-3/2) transition as detected signal in the $\nu_{2}$ dimension, and since phase is transferred between the two coherences, elaborate schemes for obtaining quadrature information in the $\nu_{1}$ dimension are unnecessary. However, it has one overriding disadvantage. As can be seen in the SCOSY spectrum of $\mathrm{SbCl}_{3}$ in Fig. 6(b), obtained with the identical sample and conditions as used in the previous experiment, the two-dimensional spectrum exhibits "phase-twist," 14 a consequence of the transformation of the dispersive part of the first Fourier transform into absorbtive signal by the second transform. This phase twist broadens the two-dimensional signal and creates artifacts. The key to removing phase twist is to produce a "timereversed" signal ${ }^{15} \exp \left(-i \omega_{1} t_{1}\right) \exp \left(i \omega_{2} t_{2}\right)$ in the first dimension in parallel with the normal signal $\exp \left(i \omega_{1} t_{1}\right) \exp \left(i \omega_{2} t_{2}\right)$. Unfortunately, there seems no straightforward way to do this in the simple SCOSY experiment. Therefore, SCOSY appears to be largely of heuristic value.

We can modify the spinor experiment to allow observation of the evolution of the double-quantum transition. A $\pi / 2\left(\nu_{1}\right) \pi\left(\nu_{2}\right)$ pulse sequence creates double-quantum coherence, which can be allowed to evolve in the $t_{1}$ period. This transition is weakly allowed for systems without axial symmetry, but generally can be observed directly only with great difficulty. Therefore, another $\pi\left(\nu_{1}\right)$ pulse converts the double-quantum coherence to single-quantum coherence of the $\nu_{2}$ transition, which is then detected. The linewidth of the double-quantum coherence is about twice that of the singlequantum transitions. The same pulse sequence was applied in NMR to detect forbidden transitions by Hatanaka et al., ${ }^{16}$ and investigated theoretically in NQR by Reddy and Narasimhan ${ }^{17}$ and Ramamoorthy. ${ }^{18}$ Of course, the doublequantum coherence could as easily be detected via the $\nu_{1}$ single-quantum coherence, by putting the second $\pi$ pulse on $\nu_{2}$ rather than $\nu_{1}$.

\section{Dual mode coherence transfer}

The NQ-MSCOSY pulse sequence [Fig. 5(c)] was developed to attain a spectrum with the improved signal-to-noise ratio of SCOSY, but without the phase twist problem. It is a conceptual hybrid of the two earlier experiments, in which half the coherence is transferred via the longitudinal and half by the double-quantum route. As in the original NQ-COSY experiment, a $\pi / 2\left(\nu_{1}\right)-t_{1}-\pi / 2\left(\nu_{1}\right)$ sequence allows evolution of the first single-quantum coherence, and places one of the two transverse components along the $z$ direction. Instead of allowing the residual transverse to decay or be phase cycled away, however, it is transferred into single-quantum coherence of the $\nu_{2}$ transition by the pair of $\pi$ pulses used in the SCOSY experiment. (This pulse pair also inverts the longitudinally transferred component, but this can be dealt with in data processing.) A $\pi / 2$ pulse on the $\nu_{2}$ transition, if it is in phase with the coherence transferred via the doublequantum route, will leave it unaffected, but it will accomplish the transfer of the longitudinal polarization component into single-quantum coherence $\nu_{2}$, orthogonal to the singlequantum coherence transferred by the double-quantum route. Phases of the pulses can be cycled to ensure that equal amounts of both Cartesian components of the initial singlequantum coherence are transferred by each route; moreover, the phase of the longitudinal transfer pulses can be selected so as to produce quadrature in the first dimension and eliminate phase twist. The result is a complete transfer of singlequantum coherence between the two transitions, just as in SCOSY, one half taking the route followed in NQ-COSY, the other the route followed in NQ-SCOSY. A final $\pi$ refocusing pulse on the (3/2-1/2) transition is used to avoid deadtime problems.

Using a 32 step phase cycling routine, the twodimensional correlation spectrum of ${ }^{123} \mathrm{Sb}$ in antimony(III) chloride is shown in Fig. 6(c). For purposes of comparison, the spectrum was acquired under the same acquisition conditions as NQ-COSY and NQ-SCOSY spectra shown for the same material above. The spectrum has pure phase in both dimensions, however, the improvement of the signal to noise is not substantial. It is likely that the full factor of 2 improvement over NQ-COSY is not achieved largely because of the number of pulses used in the sequence: in NQR this is a particular problem, since there is no single flip angle for all spins in the sample. Nonetheless, there is some improvement, and NQ-MSCOSY sequence is an instructive example of the flexibility available in designing pulse sequences in a three-level system. Moreover, the flip-angle problem may also be ameliorated by using composite pulses. ${ }^{19}$ 

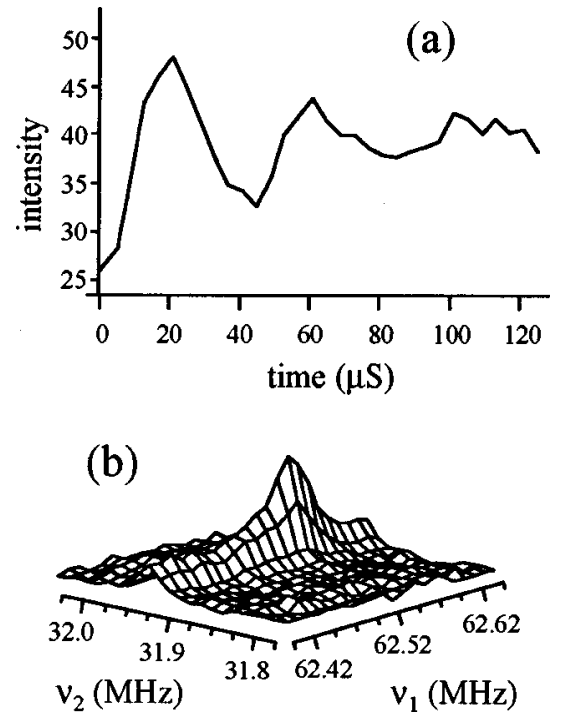

FIG. 7. (a) Intensity of the $3 / 2-1 / 2$ transition of the ${ }^{209} \mathrm{Bi}$ signal in bismuth(III) chloride, as a function of the time of nutation of the nominally forbidden 7/2-3/2 transition, obtained using the pulse sequence in Fig. 2(a). (b) The two-dimensional zero-field NMR correlation spectrum of the ${ }^{209} \mathrm{Bi}$ nucleus is bismuth(III) chloride, correlating the (7/2-3/2) and (3/2-1/2) spin transitions. In both cases the observation frequencies were $\nu_{1}=62.52 \mathrm{MHz}$ and $\nu_{2}=31.90 \mathrm{MHz}$.

\section{Indirect detection of forbidden transitions}

These experiments can be applied to the detection of nominally forbidden transitions in zero-field NMR. $\Delta m=2$ transitions, forbidden at high field and at zero field in an environment of effective axial symmetry, become weakly allowed if the asymmetry parameter is nonzero. The effect of the small transition magnetic dipole on the efficiency of detection can obviously be eliminated if the coherence is transferred to a $\Delta m=1$ transition, while the inefficiency of excitation can be mitigated by a longer pulse. In Fig. 7 we demonstrate the effect, using the spin $9 / 2{ }^{209} \mathrm{Bi}$ nucleus in bismuth(III) chloride. In this experiment, we directly excited the (7/2-3/2) double-quantum coherence with a $\pi / 2$ pulse of nominal precession frequency $21 \mathrm{kHz}$, allowed it to evolve, and then transferred it to $(3 / 2-1 / 2)$ coherence by the NQCOSY scheme for detection. Because of the large Boltzmann polarization of the 7/2-3/2 transition, a positive enhancement of the (3/2-1/2) coherence by a factor of 1.85 was observed. This enhancement is considerably lower than the theoretical value, probably as a result of inefficient irradiation of the forbidden transition. On the other hand, exciting the $(5 / 2-$ 1/2) transition and observing the connected, inner (3/2-1/2) transition, the enhancement is negative as shown in Fig. 4(b). The two-dimensional correlation spectrum of bismuth(III) chloride corresponding to the nutation experiment in Fig. 7(a) is shown in Fig. 7(b); clearly, the nearly forbidden $\Delta m=2$ transition is efficiently detected by this means.

\section{Utility}

The new pulse sequences described in this paper are of obvious utility for correlation spectroscopy at zero field, for example, for precise simultaneous measurement of transition frequencies with the elimination of correlated inhomoge- neous broadening, ${ }^{6}$ and for the assignment of complex NQR spectra. ${ }^{4}$ While phase twist is an obvious drawback of the spinor COSY sequence, this will not be an issue if phase quadrature in the first dimension is not desired, and the resulting simplicity and considerable improvement in signal to noise are significant advantages of the method. While the MS-COSY sequence in practice does not offer the full twofold signal-to-noise improvement of S-COSY, it is an enhancement of the original NQCOSY sequence, and it is likely amenable to further improvement by the use of composite pulses.

\section{CONCLUSIONS}

We have successfully applied the double resonance nutation methods to demonstrate the spin dynamics of a halfinteger high-spin quadrupolar nucleus. We have also successfully applied these two-dimensional methods to correlate connected single-quantum transitions $(\Delta m=1)$ in spin $5 / 2$ $\left({ }^{127} \mathrm{I},{ }^{121} \mathrm{Sb}\right), 7 / 2\left({ }^{123} \mathrm{Sb}\right)$ and $9 / 2\left({ }^{209} \mathrm{Bi}\right)$ systems. These correlation methods not only measure the connected spin transitions simultaneously but also eliminate inhomogeneous line broadening. Finally, we have shown that the doublequantum transitions $(\Delta m=2)$ can also be observed via the connected single-quantum transitions $(\Delta m=1)$ using the two-dimensional correlation methods.

\section{ACKNOWLEDGMENTS}

The authors thank Adilgiry Kusov and Raju Subramanian for informative discussions. This research was supported by NSF under Grant No. MCB-9604521.

\section{APPENDIX: DENSITY MATRIX APPROACH FOR THE EVALUATION OF SPIN DYNAMICS FOR SPIN 5/2 IN AN ENVIRONMENT OF AXIAL SYMMETRY}

In the absence of a fixed magnetic field, the density matrix of a half-integer spin nucleus has a twofold redundancy which makes the sign of the quantum number $m$ unimportant. ${ }^{20}$ Thus, the dynamics of a spin $5 / 2$ with axial symmetry can be evaluated as a $3 \times 3$ matrix. The thermal equilibrium reduced density matrix can be written as

$$
\rho(0)=\left[\begin{array}{ccc}
5 & 0 & 0 \\
0 & -1 & 0 \\
0 & 0 & -4
\end{array}\right]
$$

in which the rows and columns of the matrix correspond to the eigenvectors, in the order $m= \pm 5 / 2 ; \pm 3 / 2 ; \pm 1 / 2$. For an axially symmetric system, the resonance frequencies of the $(5 / 2-3 / 2)$ and (3/2-1/2) spin transitions are given by $2 \omega_{Q}$ and $\omega_{Q}$, respectively. To evaluate the pulse response in this system, radio-frequency irradiation at nutation frequencies $\omega_{1}$ and $\omega_{2}$ are set at frequencies close to $2 \omega_{Q}$ and $\omega_{Q}$, respectively. The radio-frequency pulses are denoted as $\theta(\phi)$, where $\theta_{1,2}=\omega_{1,2} t$ indicates the flip angle of the first or second resonance frequency channel and $\phi$ is the phase of the pulsed radio frequency. The responses for a two-pulse sequence at the same transition or at two connected transitions can be calculated using single transition operators. ${ }^{21}$ 
The pulse propagators for the (5/2-3/2) transition and the $(3 / 2-1 / 2)$ transition can respectively be written as

$$
\begin{aligned}
& U_{1}(\theta, \phi)=\left[\begin{array}{ccc}
\cos \frac{\theta}{2} & -i e^{-i \phi} \sin \frac{\theta}{2} & 0 \\
-i e^{i \phi} \sin \frac{\theta}{2} & \cos \frac{\theta}{2} & 0 \\
0 & 0 & 1
\end{array}\right] \\
& U_{2}(\theta, \phi)=\left[\begin{array}{cccc}
1 & 0 & 0 & \theta \\
0 & \cos \frac{\theta}{2} & -i e^{-i \phi} \sin \frac{\theta}{2} \\
0 & -i e^{i \phi} \sin \frac{\theta}{2} & \cos \frac{\theta}{2}
\end{array}\right]
\end{aligned}
$$

and evolution via the quadrupolar Hamiltonian is expressed by the propagator

$$
U_{Q}\left(\omega_{Q}, t\right)=\left[\begin{array}{ccc}
e^{-i 5 \omega_{Q} t / 3} & 0 & 0 \\
0 & e^{i \omega} Q^{t / 3} & 0 \\
0 & 0 & e^{i 4 \omega} Q^{t / 3}
\end{array}\right] .
$$

As an example, we evaluate the result of a two-pulse experiment similar to the first half of NQ-COSY. After a single pulse followed by a period of evolution, $\theta_{1}\left(\phi_{1}\right)$ $-t_{1}$, the density matrix can be evaluated as

$$
\begin{aligned}
\rho(1) & =U_{Q}\left(\omega_{Q}, t\right) U_{1}(\theta, \phi) \rho(0) U_{1}(\theta, \phi)^{-1} U_{Q}\left(\omega_{Q}, t\right)^{-1} \\
& =\left[\begin{array}{ccc}
2+3 \cos \theta_{1} & 3 i \sin \theta_{1} e^{-i \alpha} & 0 \\
-3 i \sin \theta_{1} e^{i \alpha} & 2-3 \cos \theta_{1} & 0 \\
0 & 0 & -4
\end{array}\right],
\end{aligned}
$$

where $\alpha=\phi_{1}+2 \omega_{Q} t_{1}$ is the transverse phase. After a second pulse on the (5/2-3/2 transition) pulse, the density matrix can be evaluated as

$$
\begin{aligned}
\rho(2) & =U_{1}\left(\theta_{2}, \phi_{2}\right) \rho(1) U_{1}\left(\theta_{2}, \phi_{2}\right)^{-1} \\
& =\left[\begin{array}{ccc}
S_{11} & S_{12} & 0 \\
S_{21} & S_{22} & 0 \\
0 & 0 & -4
\end{array}\right]
\end{aligned}
$$

with

$$
\begin{aligned}
& S_{11}=2+3 \cos \theta_{1} \cos \theta_{2}-\frac{3}{2} \sin \theta_{1} \sin \theta_{2}\left(e^{i \beta}+e^{-i \beta}\right) ; \\
& S_{21}=S_{12}^{*}=-\frac{3 i}{2} \sin \theta_{1}\left[\left(e^{i \alpha}-e^{-i \gamma}\right)+\left(e^{i \alpha}+e^{-i \alpha}\right) \cos \theta_{2}\right] \\
& \quad-3 i \cos \theta_{1} \sin \theta_{2} e^{i \phi_{2}} ; \\
& S_{22}=2-3 \cos \theta_{1} \cos \theta_{2}+\frac{3}{2} \sin \theta_{1} \sin \theta_{2}\left(e^{i \beta}+e^{-i \beta}\right) ;
\end{aligned}
$$

where $\alpha=\phi_{1}+2 \omega_{Q} t_{1}, \quad \beta=\phi_{1}-\phi_{2}+2 \omega_{Q} t_{1}$ and $\gamma=\phi_{1}$ $-2 \phi_{2}+2 \omega_{Q} t_{1}$. From the matrix elements shown above, after a pair of $\pi / 2$ pulses the population of the $3 / 2$ state will be proportional to $\cos \beta$. As a result, the population difference between the $\pm 3 / 2$ and $\pm 1 / 2$ states depends on the phase $\beta$, which contains information about the evolution of the $(5 / 2-$ $3 / 2$ ) transition during the $t_{1}$ period.

The further propagation of this experiment, and of other experiments discussed in this paper, are left as an exercise for the reader.

${ }^{1}$ R. Ramachandran and E. Oldfield, J. Chem. Phys. 80, 674 (1984).

${ }^{2}$ G. S. Harbison, A. Slokenbergs, and T. M. Barbara, J. Chem. Phys. 90, 5292 (1989).

${ }^{3}$ G. S. Harbison and A. Slokenbergs, Z. Naturforsch. 45A, 575 (1990).

${ }^{4}$ M.-Y. Liao, R. Templin, and G. S. Harbison, J. Am. Chem. Soc. 117, 9535 (1995)

${ }^{5}$ M.-Y. Liao and G. S. Harbison, J. Magn. Reson. 99, 198 (1992).

${ }^{6}$ M.-Y. Liao and G. S. Harbison, J. Chem. Phys. 100, 1895 (1994).

${ }^{7}$ T. C. Wang, Phys. Rev. 99, 566 (1955).

${ }^{8}$ H. G. Robinson, Phys. Rev. 100, 1731 (1955).

${ }^{9}$ M. Bloom, E. L. Hahn, and B. Herzog, Phys. Rev. 97, 1699 (1955).

${ }^{10}$ D. J. States, R. A. Haberkorn, and D. J. Ruben, J. Magn. Reson. 48, 286 (1982).

${ }^{11}$ L. D. Landau and E. M. Lifshitz, in Quantum Mechanics (Non-relativistic Theory), 3rd revised ed. (Pergamon, Oxford, 1977), pp. 204-224.

${ }^{12}$ M. E. Stoll, A. J. Vega, and R. W. Vaughan, Phys. Rev. A 16, 1521 (1977).

${ }^{13}$ M. Mehring, P. Hoefer, A. Grupp, and H. Seidel, Phys. Lett. A 106, 146 (1984).

${ }^{14}$ G. Bodenhausen, R. Freeman, R. Niedermayer, and D. L. Turner, J. Magn. Reson. 26, 133 (1977).

${ }^{15}$ A. Hagemeyer, K. Schmidt-Rohr, and H. W. Spiess, Adv. Magn. Reson. 13, 85 (1989).

${ }^{16}$ H. Hatanaka and T. Hashi, J. Phys. Soc. Jpn. 39, 1139 (1975).

${ }^{17}$ R. Reddy and P. T. Narasimhan, J. Mol. Struct. 192, 309 (1989).

${ }^{18}$ A. Ramamoorthy, Mol. Phys. 72, 1425 (1991).

${ }^{19}$ A. Ramamoorthy and P. T. Narasimhan, J. Mol. Struct. 192, 333 (1989).

${ }^{20}$ M. Goldman, Adv. Magn. Reson. 14, 59 (1990).

${ }^{21}$ S. Vega, J. Chem. Phys. 68, 5518 (1978). 